Témoigner Témoigner. Entre histoire et mémoire

Getuigen Revue pluridisciplinaire de la Fondation Auschwitz

$122 \mid 2016$

Révisionisme et négationisme

Le centre de mise à mort de Sobibór. Le secret de la technique de mise à mort à Sobibór mis à jour

Moordcentrum Sobibor: de techniek van de dood ontrafeld

Johan Puttemans

Traducteur : Anneleen Spiessens

(2) OpenEdition

Journals

Édition électronique

URL : https://journals.openedition.org/temoigner/4104

DOI : $10.4000 /$ temoigner.4104

ISSN : 2506-6390

Éditeur :

Éditions du Centre d'études et de documentation Mémoire d'Auschwitz, Éditions Kimé

Édition imprimée

Date de publication : 2 mai 2016

Pagination : $38-45$

ISSN : 2031-4183

Référence électronique

Johan Puttemans, « Le centre de mise à mort de Sobibór. Le secret de la technique de mise à mort à Sobibór mis à jour », Témoigner. Entre histoire et mémoire [En ligne], 122 | 2016, mis en ligne le 30 septembre 2021, consulté le 04 janvier 2022. URL : http://journals.openedition.org/temoigner/4104 ; DOI : https://doi.org/10.4000/temoigner.4104 

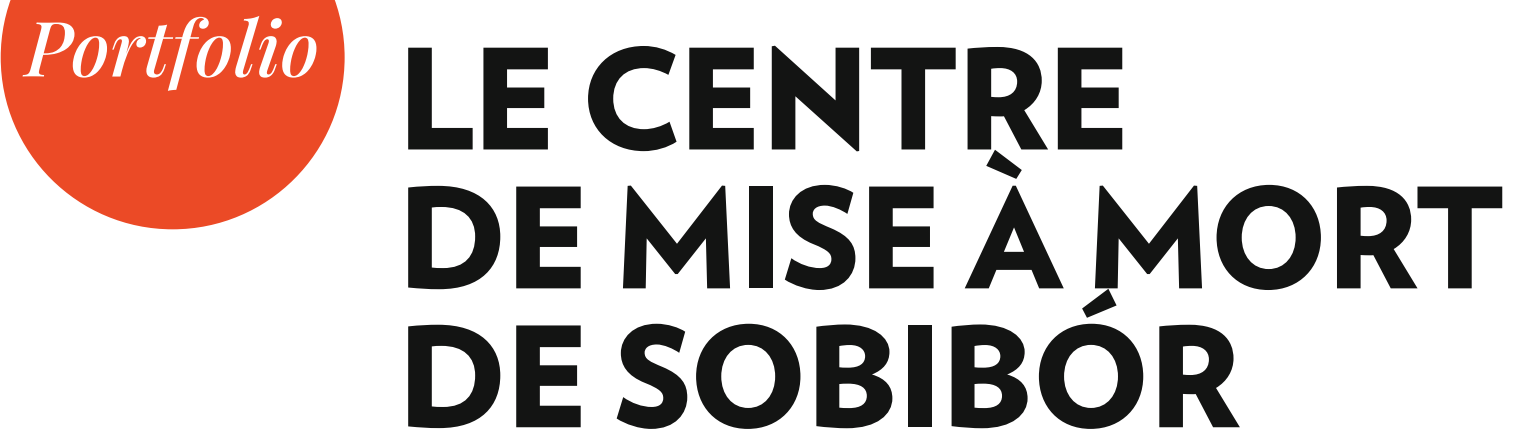

\section{Le secret de la technique de mise à mort à Sobibór mis à jour.}

\footnotetext{
$\rightarrow$ G.B. $=$

(C) Georges Boschloos -

Fondation Auschwitz

J.P. =

(C) Johan Puttemans -

Fondation Auschwitz
}

(1) Décidé lors de la conférence secrète du Wannsee le 20 janvier 1942.

Source :

http://www.deathcamps.org/reinhard/wannsee/text.html

(2) Terme renvoyant au titre de l'ouvrage du chercheur français Patrick Desbois.

(3) Allusion à l'extermination des Juifs voulue par le Gouvernement général et le District de Bialystok. a Deuxième République polonaise fondée après la Première Guerre mondiale a été, une fois conquise par l'Allemagne nazie et l'URSS, divisée entre ces deux États dictatoriaux. L'ouest du pays fut intégré à l'Allemagne hitlérienne, tandis que l'Union soviétique de Staline envahit l'est. La partie centrale devint alors un État tampon entre ces grandes puissances agressives. Pendant la Seconde Guerre mondiale, la Pologne centrale, devenue Generalgouvernement [gouvernement général], est placée sous l’administration du juriste nazi Hans Frank; elle fonctionne comme un État vassal où les ordres émanent directement de Berlin. Notons que par cette conquête et l'intégration officieuse de la Pologne centrale, le pouvoir antisémite et raciste avait la mainmise non seulement sur des Untermenschen slaves, mais aussi sur près de 2284000 Juifs $^{1}$. Quand, à l'automne 1941, Hitler décide d'exterminer tous les Juifs d'Europe, l'importante communauté juive de l'État vassal pose donc aussi problème.

La Shoah par balles ${ }^{2}$ menée dans les régions orientales occupées semble irréalisable dans le Gouvernement général. Trois lieux de la mort y sont donc aménagés. En mars 1942, le premier centre d'extermination de l' «Aktion Reinhardt $»^{3}$ ouvre à Bełżec. Le système des centres de mise à mort est agrandi un mois plus tard avec la construction d'un deuxième site, près du village polonais de Sobibór. Cette région marécageuse et densément boisée, située à $5 \mathrm{~km}$ de la rivière Bug, marque la frontière entre le gouvernement général et le Reichskommissariat d'Ukraine. Les nazis peuvent y exterminer la population juive sans que l'on y prête attention.

Pour optimiser la «capacité» de ces centres d'extermination, des travaux d'agrandissement sont réalisés. Fin juillet 1942, l'ancienne chambre à gaz de Sobibór est remplacée par un nouveau bâtiment en pierre. Les travaux sont dirigés par le $S S$ Unterscharführer [sergent SS] Erwin Lambert. Du printemps 1942 à l'automne 1943, 




entre 170000 et 250000 Juifs y seront assassinés juste après leur arrivée.

Après la rébellion de Sobibór, le 14 octobre 1943, une cinquantaine de Juifs survit au centre d'extermination. Ce sont des Arbeitsjuden, des travailleurs forcés juifs chargés de trier les biens des Juifs gazés. Parmi eux, Thomas Blatt, décédé en Avril 2016. Durant l'automne 1943, les traces du génocide sont effacées sur ordre de Heinrich Himmler, commandant en chef des SS. À l'aide de témoignages tant de survivants juifs que de gardiens SS présents sur le site, on a toutefois pu obtenir un aperçu du fonctionnement du centre et on est parvenu à esquisser un schéma des lieux grossier, qui prête parfois à discussion.

En 2000 et 2001, le prof. dr. Andrzej Koła, historien etarchéologue polonais, a réalisé des fouilles à Sobibór. S’il a réussi à localiser plusieurs fosses communes, il restait encore difficile d'avoir une image globale du centre d'extermination. L'archéologue israélien Yoram Haimi, qui, depuis 2007, organise chaque année des fouilles avec son collègue polonais Wojchiech Mazurek, fit néanmoins plusieurs découvertes intéressantes : mise à jour de la double enceinte en 2010, de la Himmelfahrtstraße (chemin menant aux chambres à gaz) de $240 \mathrm{~m}$ de long en 2011 et exhumation d'objets ayant appartenu aux victimes en 2013. Ce n'est qu'en septembre 2014 qu'est faite une découverte majeure : les archéologues retrouvent les fondations en brique des chambres à gaz, quatre pièces et un couloir, abrités par le seul bâtiment en pierre du centre d'extermination. Toutes ces découvertes seront intégrées au nouveau mémorial. Le centre de mise à mort de Sobibór devrait dévoiler encore d'autres secrets à l'avenir. I

Johan Puttemans

Mémoire d'Auschwitz ASBL

Traduit du néerlandais par Anneleen Spiessens
Emplacement des fouilles archéologiques. La colonne en pierre symbolise les chambres à gaz. Élevée en 1965 par le sculpteur polonais Mieczysław Welter. 
$\rightarrow$ Emplacement des fouilles archéologiques. Le monument au premier plan représente une femme et un enfant assassinés dans les chambres à gaz.

Érigé en 1965 par le sculpteur polonais Mieczysław Welter. Ce monument a été déplacé au milieu des années 1980.

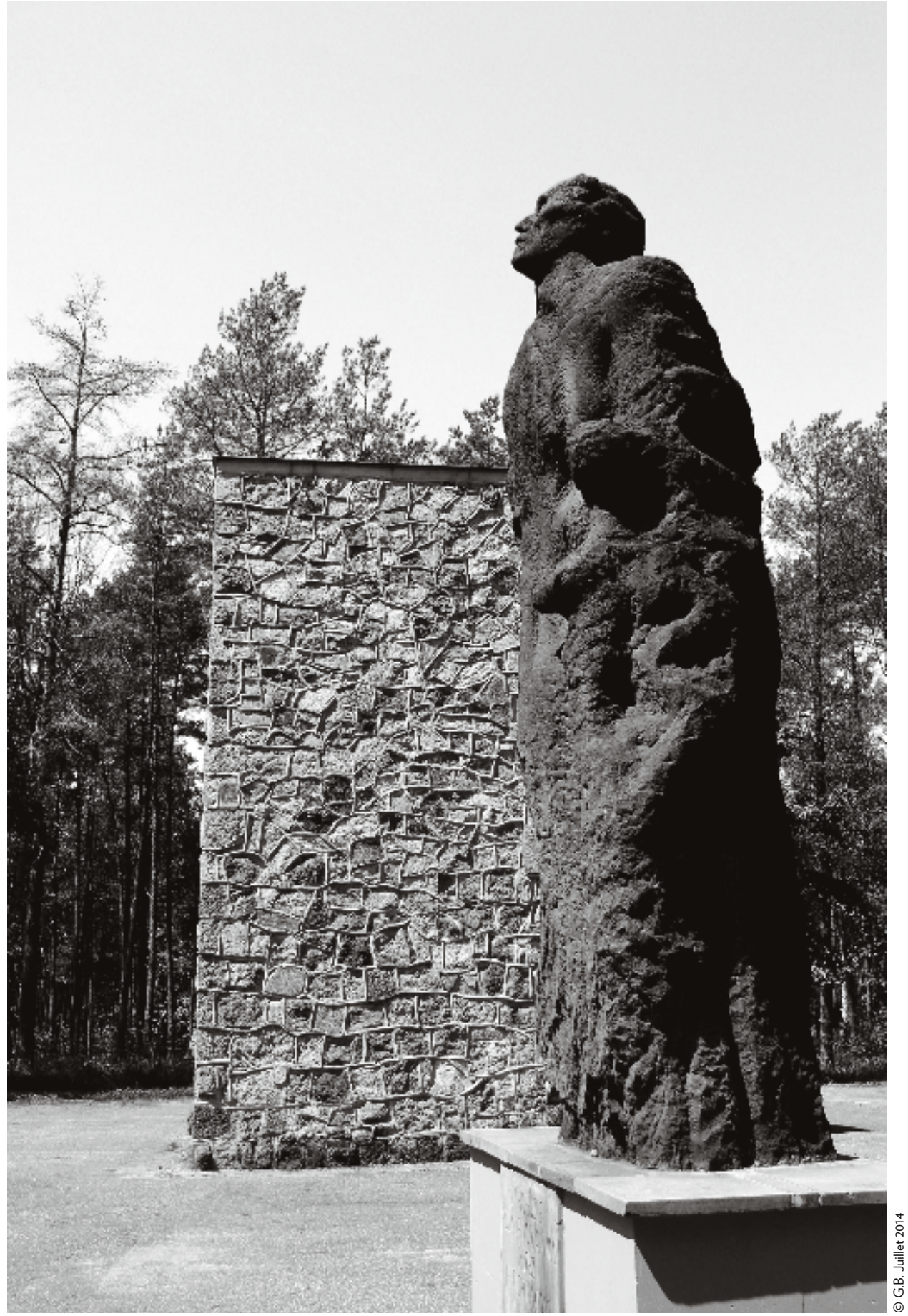



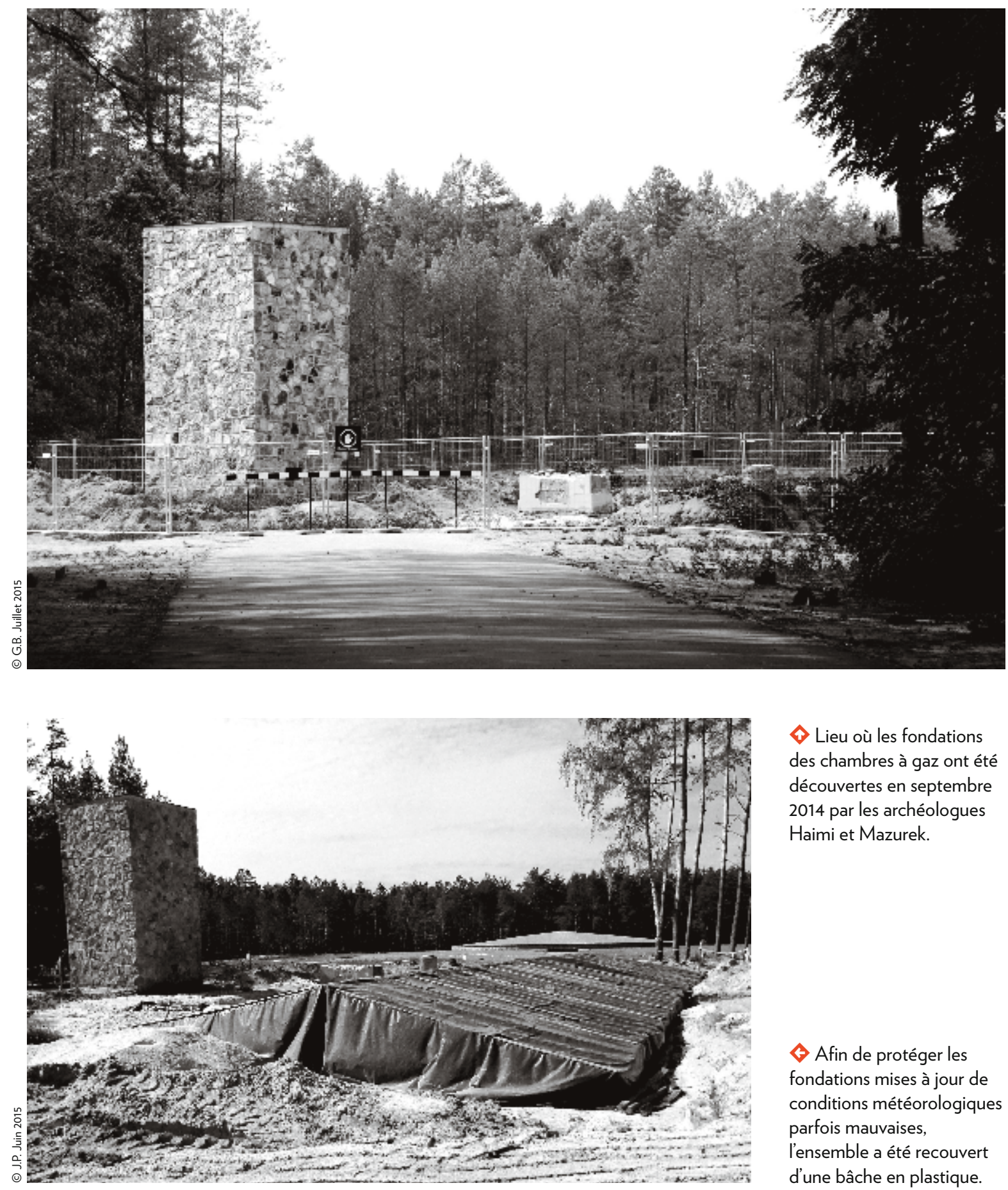

- Lieu où les fondations des chambres à gaz ont été découvertes en septembre 2014 par les archéologues Haimi et Mazurek.

$\diamond$ Afin de protéger les fondations mises à jour de conditions météorologiques parfois mauvaises, l'ensemble a été recouvert d'une bâche en plastique. 


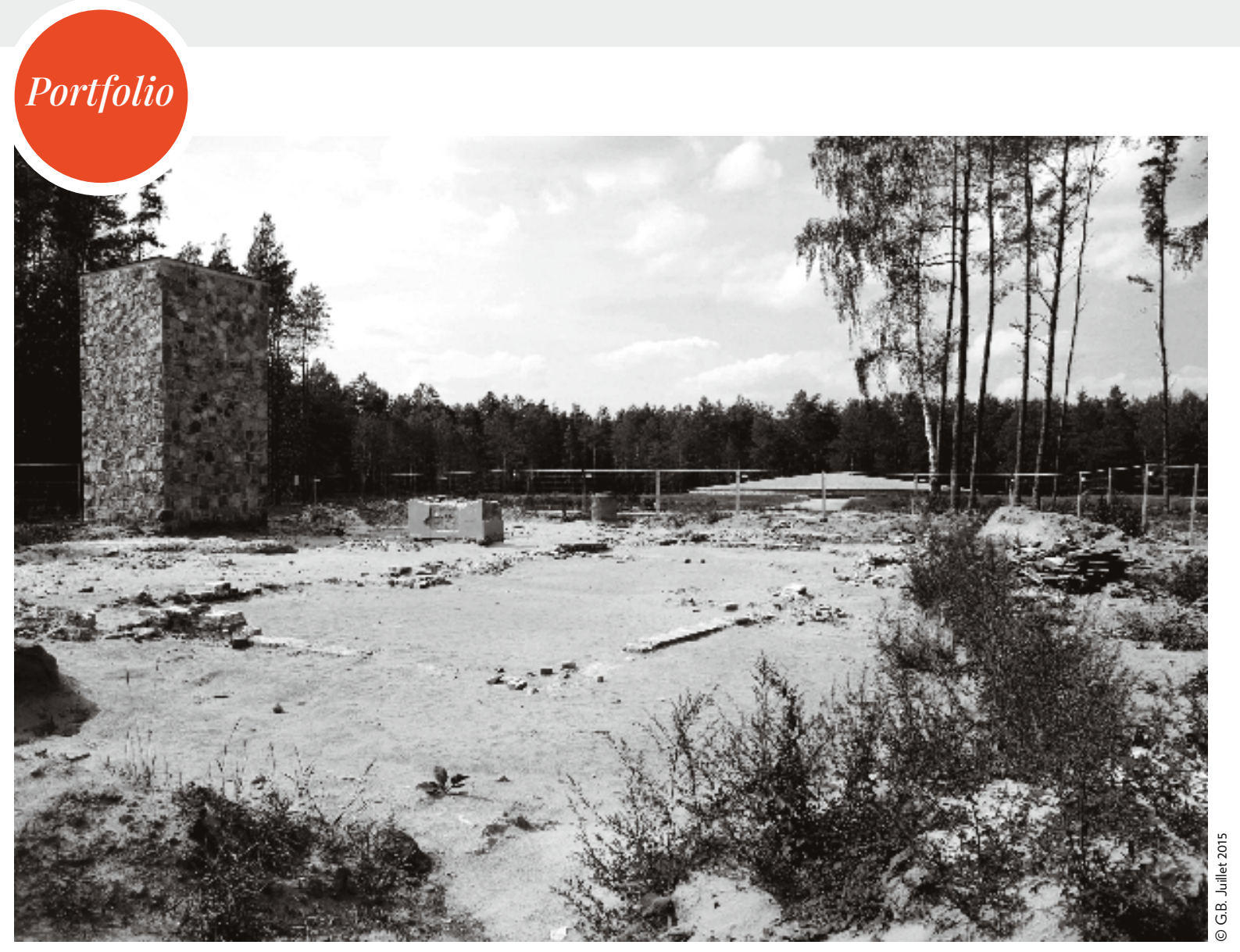

ᄂ Le revêtement de béton retiré dévoile les fondations des chambres à gaz du centre de mise à mort de Sobibór.

$\rightarrow$ Les fondations

clairement reconnaissables des chambres à gaz construites à l'été 1942 pour accroître la capacité de mise à mort.

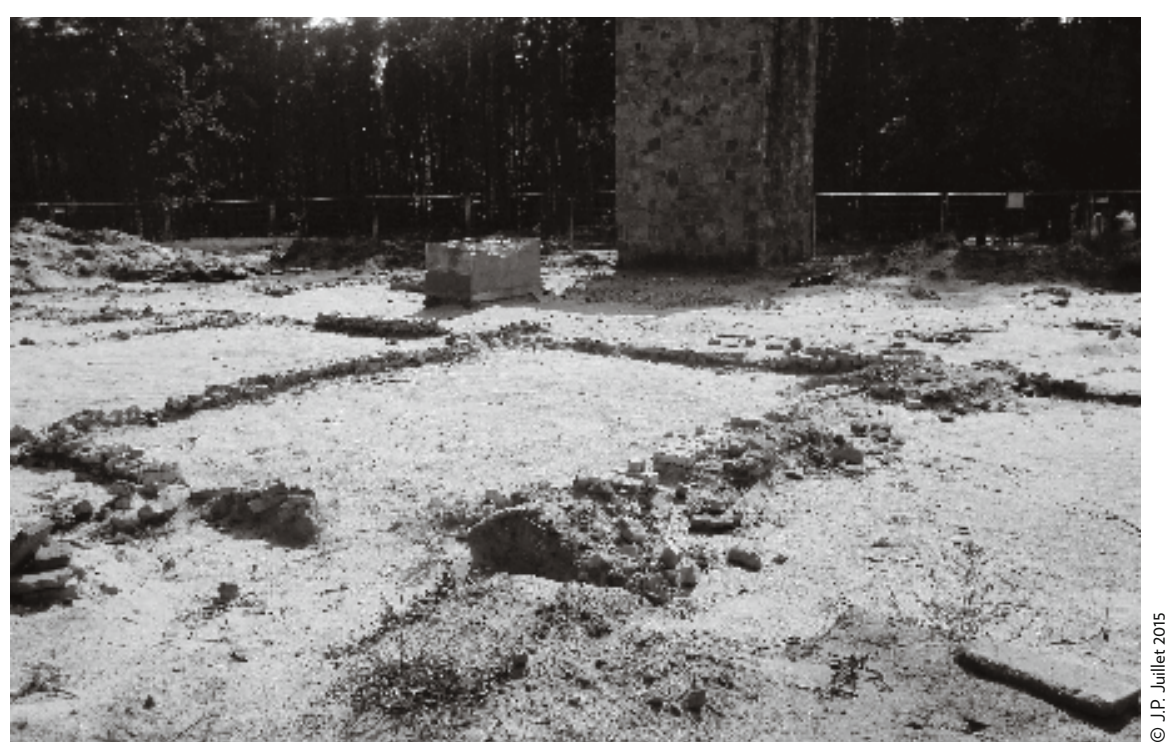



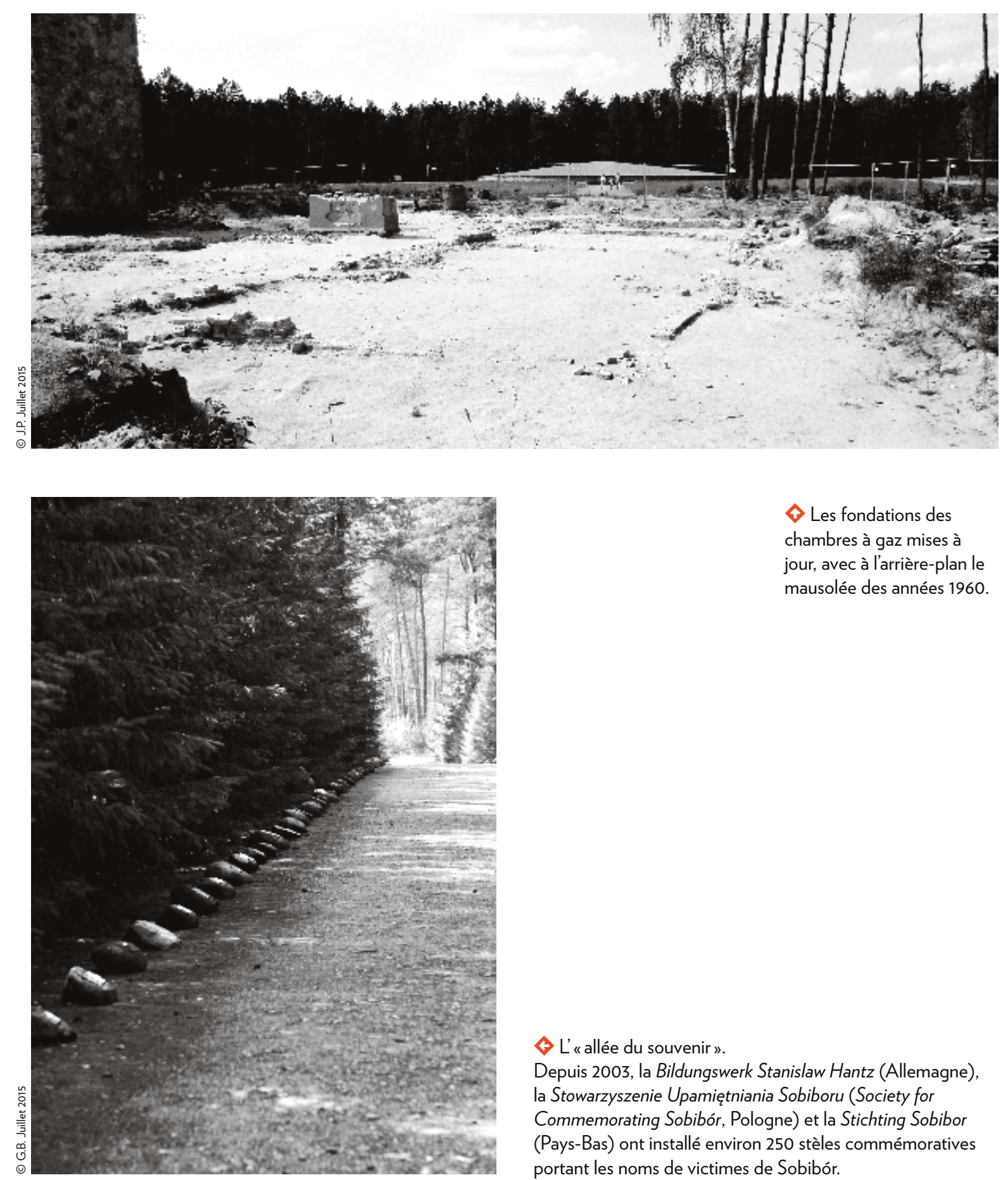

$\uparrow$ Les fondations des chambres à gaz mises à jour, avec à l'arrière-plan le mausolée des années 1960. $\diamond$ L' «allée du souvenir ».

Depuis 2003, la Bildungswerk Stanislaw Hantz (Allemagne), la Stowarzyszenie Upamiętniania Sobiboru (Society for Commemorating Sobibór, Pologne) et la Stichting Sobibor (Pays-Bas) ont installé environ 250 stèles commémoratives portant les noms de victimes de Sobibór. 


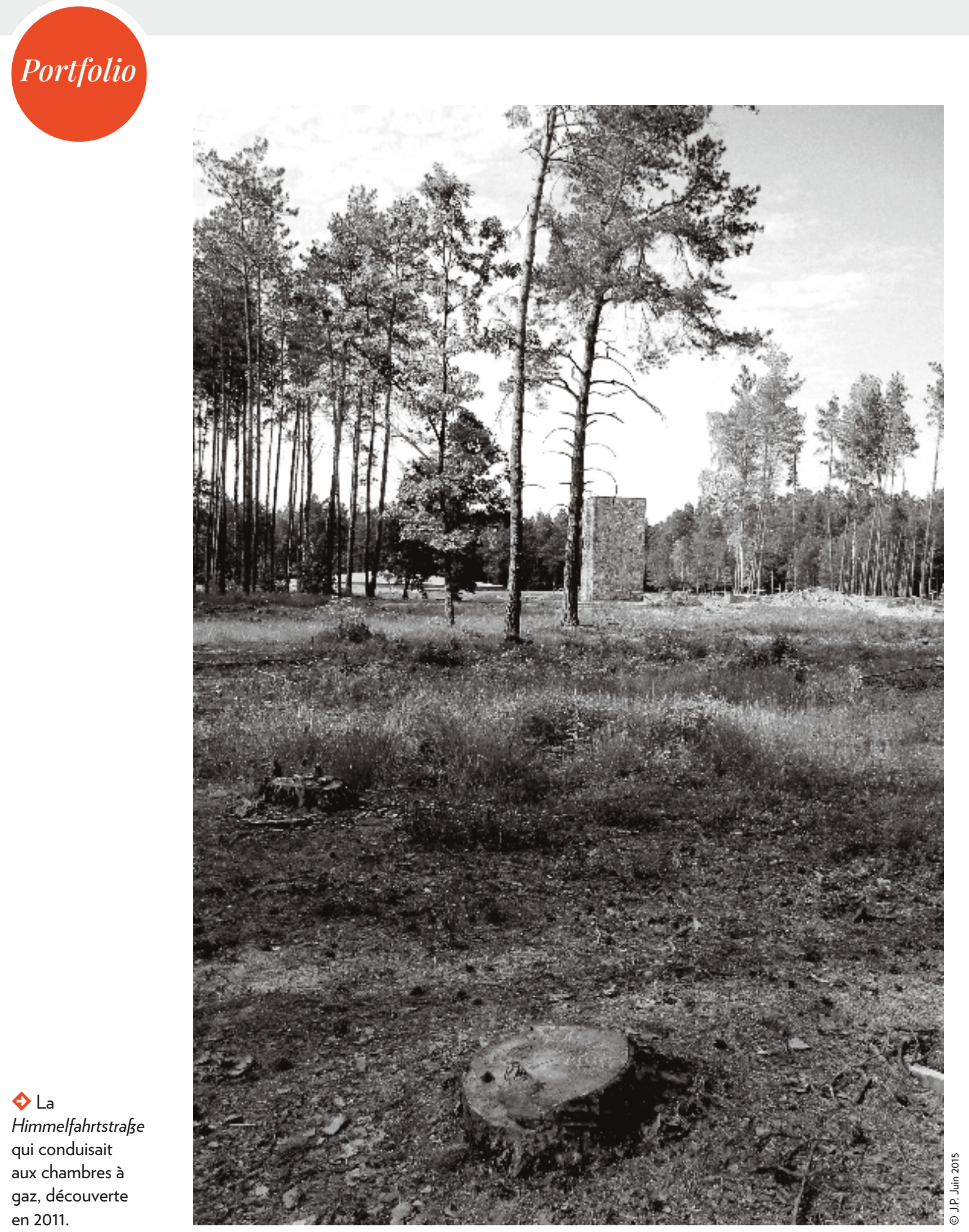




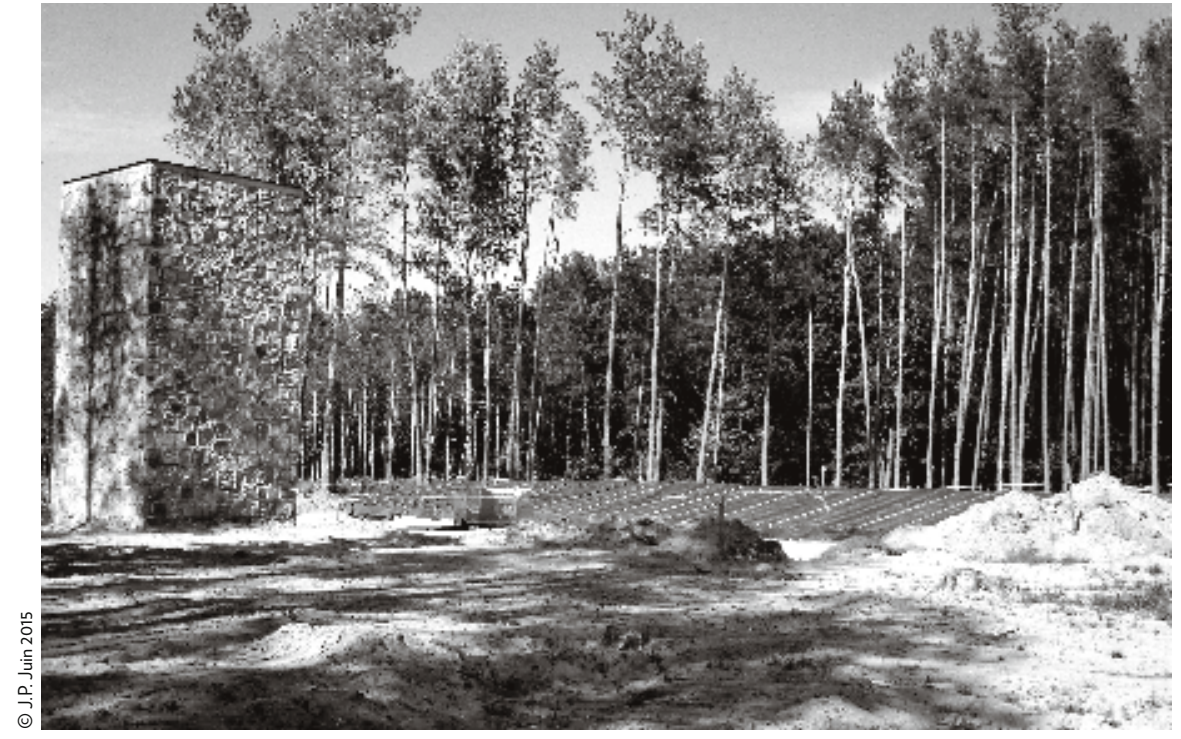

$\diamond$ Vue des fondations des chambres à gaz (ici sous une bâche en plastique) depuis la Himmelfahrtstrafze. Celle-ci sera intégrée au nouveau mémorial.

$\checkmark$ En 1965, l'artiste polonais Mieczysław Welter a érigé ce mausolée à l'endroit où les corps étaient enterrés dans les fosses, puis étaient brûlés.
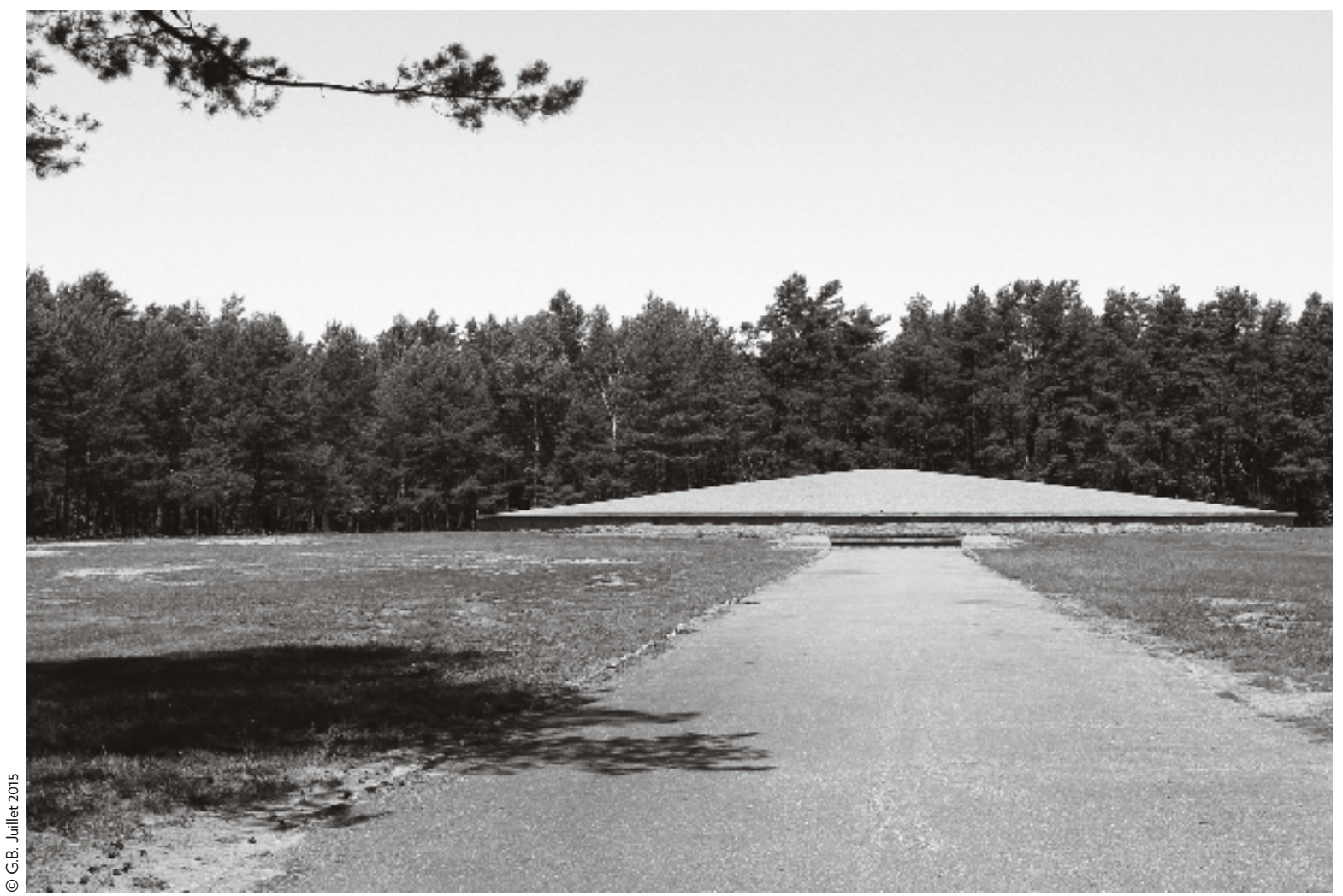